\title{
THE ARITHMETIC LOCAL NORI FUNDAMENTAL GROUP
}

\author{
MATTHIEU ROMAGNY, FABIO TONINI, LEI ZHANG
}

\begin{abstract}
In this paper we introduce the local Nori fundamental group scheme of a reduced scheme or algebraic stack over a perfect field $k$. We give particular attention to the case of fields: to any field extension $K / k$ we attach a pro-local group scheme over $k$. We show how this group has many analogies, but also some crucial differences, with the absolute Galois group. We propose two conjectures, analogous to the classical Neukirch-Uchida Theorem and Abhyankar Conjecture, providing some evidence in their favor. Finally we show that the local fundamental group of a normal variety is a quotient of the local fundamental group of an open, of its generic point (as it happens for the étale fundamental group) and even of any smooth neighborhood.
\end{abstract}

\section{INTRODUCTION}

Let $k$ be a perfect field of characteristic $p \geqslant 0$. Given a $k$-variety $X$ with a rational point $x \in X(k)$, Nori constructed a profinite group scheme $\pi^{\mathrm{N}}(X, x)$ satisfying the following universal property: there exists a natural bijection

$$
\operatorname{Hom}_{k}\left(\pi^{\mathrm{N}}(X, x), G\right) \longrightarrow\{\text { pointed } G \text {-torsors }(P, p) \rightarrow(X, x)\}
$$

for all (pro-)finite group schemes $G$ over $k$. This was later called the Nori fundamental group of $X$ at $x$. Since every finite group scheme is an extension of an étale group scheme by a local one (recall that a group scheme is called local, or infinitesimal, if it is finite and connected), it is also interesting to focus on both subclasses. One finds that the maximal pro-étale quotient $\pi^{\mathrm{N}, \text { ét }}(X, x)$ and the maximal pro-local quotient $\pi^{\mathrm{L}}(X, x)$ of Nori's fundamental group satisfy the universal properties restricted to the subclasses.

In the same way as Grothendieck's étale fundamental group "parametrizes" Galois étale covers, Nori's fundamental group "parametrizes" pointed torsors under finite group schemes. One important difference is the necessity to label the torsors with a base point in Nori's version. This has regrettable consequences: for instance $\pi^{\mathrm{N}}(\operatorname{Spec} k, x)=1$ even if $k$ is not algebraically closed. One of the purposes of the present paper is to remove the need for a rational point. For any reduced scheme or algebraic stack $X$ over $k$ we will define the local Nori fundamental group scheme $\pi^{\mathrm{L}}(X / k)$, a pro-local group scheme over $k$ with bijections

Date: June 23, 2021.

This work was supported by the Research Grants Council (RGC) of the Hongkong SAR China (Project No. CUHK 14301019). The second author was supported by GNSAGA of INdAM. The first author was supported by the Centre Henri Lebesgue, program ANR-11-LABX-0020-01 and would like to thank the executive and administrative staff of IRMAR and of the Centre Henri Lebesgue for creating an attractive mathematical environment. 
functorial in the (pro)-local group scheme $G$ :

$$
\operatorname{Hom}_{k}\left(\pi^{\mathrm{L}}(X / k), G\right) \longrightarrow\{G \text {-torsors } P \rightarrow X\} .
$$

This definition makes sense because the category of torsors under local group schemes is equivalent to a set, that is it is a groupoid with only identities as automorphisms, unlike what happens for general finite group schemes.

The emancipation from the need of a rational point in the theory of Nori's fundamental group was also addressed by Borne and Vistoli [BV15]. Their idea was to use finite gerbes instead of finite group schemes. The so-called Nori fundamental gerbe thus obtained recovers the Nori fundamental group as soon as one fixes a rational point. In [TZ19, §7] the same theory was worked out for local gerbes, obtaining the local Nori fundamental gerbe (see 2.2). It turns out that the gerbe "is exactly" the local Nori fundamental group without the need of any point, because a local gerbe over a perfect field is uniquely and therefore canonically neutral. This is actually how we come up with the above definition (see 2.7). Moreover, as a consequence of [TZ19] there is an explicit Tannakian description of the representation category $\operatorname{Rep} \pi^{\mathrm{L}}(X / k)$ in terms of vector bundles on $X$ (see 2.4).

$$
\therefore
$$

As an evidence that the local Nori fundamental group has valuable arithmetic content, we shall see that it is an extremely interesting object when $X$ is the spectrum of a field $K$. In this case, we use the simplified notation

$$
\pi^{\mathrm{L}}(K / k):=\pi^{\mathrm{L}}(\operatorname{Spec} K / k) .
$$

Due to the parallel between the local Nori fundamental group of a field $K$ and its absolute Galois group, it is desirable to see to what extent their behaviours resemble or differ. We approach the question from three viewpoints: the Galois correspondence, the anabelian philosophy, and the inverse Galois problem. In the rest of the introduction, we present our findings: two main results and two conjectures with piece of evidence supporting them. Our first main theorem is a part of the Galois correspondence.

Theorem I. Let $k$ be a perfect field and $K / k$ a field extension. Denote by $\mathrm{PI}(K)$ the totally ordered set of purely inseparable extensions of $K$. The mapping

$$
\begin{aligned}
\mathrm{PI}(K) & \longrightarrow\left\{\text { subgroups of } \pi^{\mathrm{L}}(K / k)\right\} \\
E / K & \longrightarrow\left(\pi^{\mathrm{L}}(E / k) \rightarrow \pi^{\mathrm{L}}(K / k)\right) .
\end{aligned}
$$

is well-defined and an order-reversing embedding, that is $\pi^{\mathrm{L}}\left(E_{1} / k\right) \subseteq \pi^{\mathrm{L}}\left(E_{2} / k\right) \subseteq \pi^{\mathrm{L}}(K / k)$ if and only if $K \subseteq E_{2} \subseteq E_{1}$ for all $E_{1}, E_{2} \in \mathrm{PI}(K)$.

It would be interesting to understand the relation between the above embedding and previous classifications of purely inseparable extensions, starting from the Jacobson correspondence and its early developments (see [Ja44], [Ge64], [GZ70], [Ch71], [He71], [Mo75]) until very recent generalizations (see [Ba18] and [BW20]).

We also show that the role played in Galois Theory by separably closed fields is played in the theory of the local Nori fundamental group by perfect fields, in the precise sense 
that $K$ is perfect if and only if $\pi^{\mathrm{L}}(K / k)=1$ (see 1.4). However, exceeding enthusiasm for the desired analogy should not reign. Indeed, we prove that the map in Theorem $I$ is highly non surjective unless $K$ is perfect. Moreover, for a nontrivial finite purely inseparable extension $L / K$ the subgroup $\pi^{\mathrm{L}}(L / k)$ never has finite index in $\pi^{\mathrm{L}}(K / k)$ (see 1.6).

Nevertheless, we expect $\pi^{\mathrm{L}}(E / k)$ to carry a lot of information on the field $E$ and in some cases to be able to recover $E$. We propose the following anabelian-style conjecture.

Conjecture I. Let $k$ be a perfect field and $K, E$ finitely generated field extensions of $k$. Assume that $K$ and $E$ are not finite over $k$. Then

$$
\pi^{\mathrm{L}}(K / k) \simeq \pi^{\mathrm{L}}(E / k) \Longrightarrow K \simeq E
$$

(isomorphism of $k$-group schemes on the left, isomorphism of $k$-extensions on the right).

This is the local analogue of (a part of) the Neukirch-Uchida Theorem:

Theorem ([Uch77], [Pop94], [Pop02], [Mo99]). If K, E are number fields, then

$$
\operatorname{Gal}(K) \simeq \operatorname{Gal}(E) \Longrightarrow K \simeq E .
$$

If $K, E$ are infinite and finitely generated fields over $\mathbb{F}_{p}$ then

$$
\operatorname{Gal}(K) \simeq \operatorname{Gal}(E) \Longrightarrow K^{\text {perf }} \simeq E^{\text {perf }}
$$

where $(-)^{\text {perf }}$ denotes the perfect closure functor of fields.

Theorem I aims to be a baby case of Conjecture I. More generally we show that, in the hypothesis of Conjecture I, a $k$-map $K \rightarrow E$ induces an isomorphism $\pi^{\mathrm{L}}(E / k) \rightarrow \pi^{\mathrm{L}}(K / k)$ if and only if it is an isomorphism (see 2.19). The map $\pi^{\mathrm{L}}(E / k) \rightarrow \pi^{\mathrm{L}}(K / k)$ is actually a quotient for separable extensions $E / K$ (see 3.5). The reason why we expect Conjecture I to be true is that the explicit description of $\operatorname{Rep} \pi^{\mathrm{L}}(K / k)$ seems to tell a lot about the arithmetic of $K$. For instance, we construct isomorphisms of groups:

$$
\operatorname{Hom}\left(\pi^{\mathrm{L}}(K / k), \mathbb{G}_{m}\right) \simeq\left(K^{\text {perf }}\right)^{*} / K^{*} \text { and } \operatorname{Hom}\left(\pi^{\mathrm{L}}(K / k), \mathbb{G}_{a}\right) \simeq K^{\text {perf }} / K .
$$

Multiplicative and additive structures on $K^{\text {perf }}$ are determined by $\pi^{\mathrm{L}}(K / k)$, yet we still see no way to relate them for the moment. The above groups can be described in terms of one-dimensional representations of $\pi^{\mathrm{L}}(K / k)$ and extensions of the trivial representation respectively. We therefore expect that crucial information is contained in higher dimensional representations.

Finally, in the spirit of the inverse Galois problem, we consider the fundamental example given by the field of rational functions in one variable, and we state the following conjecture.

Conjecture II. Let $k$ be a perfect field. Then any local group scheme $G$ over $k$ is a quotient of $\pi^{\mathrm{L}}(k(t) / k)$, that is there exists a $G$-torsor $P \rightarrow \operatorname{Spec} k(t)$ which is not induced by a torsor under a strict subgroup of $G$.

This is analogous to the "generic Abhyankar conjecture" for the absolute Galois group:

Theorem ([Har94]). Let $k$ be an algebraically closed field. Then any finite group $G$ is a quotient of $\operatorname{Gal}(k(t))$, that is there exists a Galois extension of $k(t)$ with group $G$. 
We call Conjecture II the local Abhyankar conjecture. A very similar, stronger conjecture was stated in [Ot18, Question 1.1] and proved in several cases in the same paper and in [Ot19], [OTZ20]. The setting is slightly different: for an affine $k$-curve $U$, Otabe [Ot18, Question 1.1] predicts which local group schemes over $k$ appear as a quotient of $\pi^{\mathrm{L}}(U / k)$, just like the general Abhyankar conjecture predicts which finite groups occur as a quotient of the étale fundamental group $\pi_{1}(U, u)$. Our contribution here is in proving that the relation between the global and generic local Abhyankar conjectures is similar to that in the non-local case. Namely, the generic local fundamental group surjects onto the global one. We prove more generally the following result:

Theorem II. Let $\mathcal{X}$ be a normal, quasi-separated and irreducible algebraic stack over $k$ and $\mathcal{V} \rightarrow \mathcal{X}$ a map from a reduced algebraic stack. If $\mathcal{V} \rightarrow \mathcal{X}$ has non empty reduced geometric generic fiber then the map

$$
\pi^{\mathrm{L}}(\mathcal{V} / k) \rightarrow \pi^{\mathrm{L}}(\mathcal{X} / k)
$$

is surjective. This is the case, for instance, if $\mathcal{V} \rightarrow \mathcal{X}$ is either

(1) flat, geometrically reduced and has an open image (e.g. an open embedding);

(2) it exhibits $\mathcal{V}$ as a generic point of a smooth atlas of $\mathcal{X}$.

In particular, all groups considered in [Ot18] and [OTZ20] satisfy Conjecture II. Finally we show that Conjecture II implies the same result for many other fields. Indeed we show that $\pi^{\mathrm{L}}(k((t)) / k) \rightarrow \pi^{\mathrm{L}}(k(t) / k)$ is surjective (see 3.6) and that, if $K$ is any finitely generated field extension of $k$ of positive transcendence degree, there exists an indeterminate $t \in K$ such that also $\pi^{\mathrm{L}}(K / k) \rightarrow \pi^{\mathrm{L}}(k(t) / k)$ is surjective (see 3.7).

The paper is divided as follows. In Section 1 we define the local Nori fundamental group for a field using Tannaka Theory, and we prove that Theorem I holds for this group scheme. In Section 2 we introduce the general local Nori fundamental group via the local Nori fundamental gerbe, and we prove the connection with torsors via the universal property as stated in the beginning in the introduction. Finally in Section 3 we discuss surjectivity results about the local fundamental group, and we prove Theorem II.

Acknowledgement. We would like to thank D. Tossici, S. Otabe and A. Vistoli for helpful conversations and suggestions received.

\section{The local Nori fundamental group of a Field}

Let $k$ be a perfect field of positive characteristic $p$. Given a field $K$ we will denote by $K^{\text {perf }}$ its perfect closure and by $\operatorname{Vect}(K)$ the category of finite dimensional $K$-vector spaces. The aim of this section is to give a direct definition of the local fundamental group scheme of a field extension $K / k$ that avoids torsors. Then we prove that with this definition, the conclusion of Theorem I holds. The equivalence between the two definitions, namely the verification of the universal property, is proved later (see 2.13). 
Definition 1.1. Let $K / k$ be a field extension. We define $\mathcal{D}_{\infty}(K / k)$ as the category whose objects are triples $(V, W, \psi)$, where $V \in \operatorname{Vect}(K), W \in \operatorname{Vect}(k)$ and

$$
\psi: K^{\text {perf }} \otimes_{K} V \rightarrow K^{\text {perf }} \otimes_{k} W
$$

is a $K^{\text {perf }}$-linear isomorphism. An arrow $(V, W, \psi) \rightarrow\left(V^{\prime}, W^{\prime}, \psi^{\prime}\right)$ in $\mathcal{D}_{\infty}(K / k)$ is a pair $(a, b)$ composed of a $K$-linear map $a: V \rightarrow V^{\prime}$ and a $k$-linear map $b: W \rightarrow W^{\prime}$ which are compatible with $\psi$ and $\psi^{\prime}$.

The category $\mathcal{D}_{\infty}(K / k)$ with its natural tensor product and $k$-linear structure is a neutral $k$-Tannakian category with the forgetful functor $\mathcal{D}_{\infty}(K / k) \longrightarrow \operatorname{Vect}(k)$ as the fiber functor. We define the local Nori fundamental group $\pi^{\mathrm{L}}(K / k)$ of $K / k$ as the Tannakian group scheme associated with $\mathcal{D}_{\infty}(K / k)$.

Lemma 1.2. If $L / K$ is a purely inseparable extension of fields over $k$ then the induced map of group schemes $\pi^{\mathrm{L}}(L / k) \rightarrow \pi^{\mathrm{L}}(K / k)$ is a closed embedding.

Proof. By [DM82, Prop. 2.21 (b)] it is enough to show that the pullback functor

$$
\mathcal{D}_{\infty}(K / k) \rightarrow \mathcal{D}_{\infty}(L / k)
$$

is essentially surjective. Let $(V, W, \phi) \in \mathcal{D}_{\infty}(L / k)$. Clearly there is an isomorphism $(V, W, \phi) \cong\left(L^{\oplus m}, k^{\oplus m}, \varphi\right) \in \mathcal{D}_{\infty}(L / k)$. Now consider the isomorphism

$$
\varphi:\left(L^{\text {perf }}\right)^{\oplus m} \longrightarrow\left(L^{\text {perf }}\right)^{\oplus m}
$$

Since $L / K$ is purely inseparable, we can identify $K^{\text {perf }}$ with $L^{\text {perf }}$. In this case it is easy to see that $\left(K^{\oplus m}, k^{\oplus m}, \varphi\right) \in \mathcal{D}_{\infty}(K / k)$ is sent to $\left(L^{\oplus m}, k^{\oplus m}, \varphi\right)$.

Proposition 1.3. Let $K / k$ be a field extension. Then there is a canonical isomorphism

$$
\operatorname{Pic}\left(\mathcal{D}_{\infty}(K / k)\right) \simeq \operatorname{Hom}_{k}\left(\pi^{\mathrm{L}}(K / k), \mathbb{G}_{m}\right) \simeq\left(K^{\text {perf }}\right)^{*} / K^{*} .
$$

Proof. The first isomorphism exists since both sides are the group of isomorphism classes of 1-dimensional representations of $\pi^{\mathrm{L}}(K / k)$. There is a group homomorphism

$$
\begin{aligned}
\left(K^{\text {perf }}\right)^{*} & \longrightarrow \operatorname{Pic}\left(\mathcal{D}_{\infty}(K / k)\right) \\
\phi & \longmapsto(K, k, \phi) .
\end{aligned}
$$

It is easy to see that it is surjective and that its kernel is $K^{*}$.

Proof of Theorem I. By 1.2 we see that the map in the statement is well defined. All elements of $\mathrm{PI}(K)$ has a unique embedding in $K^{\text {perf }}$. Thus we have to prove that, if $K \subseteq L_{1}, L_{2} \subseteq K^{\text {perf }}$ and $\pi^{\mathrm{L}}\left(L_{1} / k\right) \subseteq \pi^{\mathrm{L}}\left(L_{2} / k\right) \subseteq \pi^{\mathrm{L}}(K / k)$, then $L_{2} \subseteq L_{1}$.

By 1.3 and the commutative diagram of group schemes

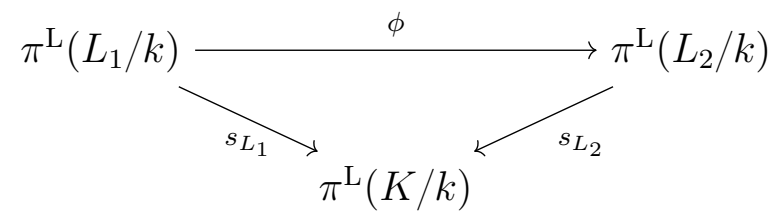


we get a commutative diagram of abelian groups

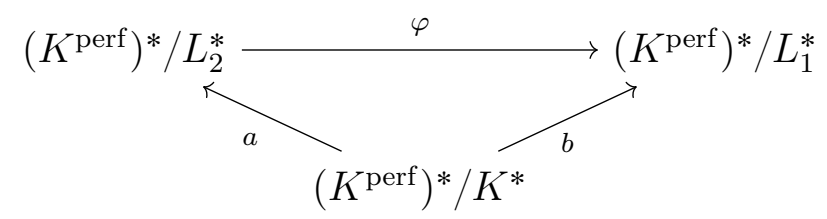

where $s_{L_{i}}$ is the inclusion $\pi^{\mathrm{L}}\left(L_{i} / k\right) \subseteq \pi^{\mathrm{L}}(K / k)$. In particular we conclude that $a$ and $b$ are induced on the perfect closure by $K \subseteq L_{2}$ and $K \subseteq L_{1}$ respectively, that is $a$ and $b$ are induced by the identity map. Then it is clear that $b\left(L_{2}^{*} / K^{*}\right)=1$, as $a\left(L_{2}^{*} / K^{*}\right)=1$. Thus we have $L_{2}^{*} / K^{*} \subseteq L_{1}^{*} / K^{*}$. This shows the inclusion $L_{2} \subseteq L_{1}$.

Corollary 1.4. Let $K / k$ be a field extension. Then $K$ is perfect if and only if $\pi^{\mathrm{L}}(K / k)=1$.

Proof. If $\pi^{\mathrm{L}}(K / k)=0$, then from Theorem I we see that $\operatorname{PI}(K)$ has just one element, that is $K$ is perfect. Now assume that $K$ is perfect, that is $K^{\text {perf }}=K$. If $(V, W, \phi) \in \mathcal{D}_{\infty}(K / k)$, then $\phi: V \longrightarrow W \otimes_{k} K$ is an isomorphism. It is easy to see that

$$
(V, W, \phi) \stackrel{(\phi, \mathrm{id})}{\longrightarrow}\left(W \otimes_{k} K, W, \mathrm{id}\right)
$$

is an isomorphism. This means that $\operatorname{Vect}(k)=\mathcal{D}_{\infty}(K / k)$, that is $\pi^{\mathrm{L}}(K / k)=1$.

Lemma 1.5. If $G$ is an affine group scheme over $k$ then there exists a canonical surjective map

$$
G \longrightarrow \mathrm{D}\left(\operatorname{Hom}\left(G, \mathbb{G}_{m}\right)\right)
$$

where $\mathrm{D}(-)$ is the diagonalizable group over $k$ associated with an abelian group, which is universal among all maps to diagonalizable group schemes.

Proof. There is a natural isomorphism

$$
\operatorname{Hom}(G, \mathrm{D}(H)) \simeq \operatorname{Hom}\left(H, \operatorname{Hom}\left(G, \mathbb{G}_{m}\right)\right)
$$

which implies the existence of a map

$$
\delta: G \rightarrow \mathrm{D}\left(\operatorname{Hom}\left(G, \mathbb{G}_{m}\right)\right)
$$

universal for maps to diagonalizable group schemes. Since $\operatorname{Im}(\delta)$ is diagonalizable and a closed subgroup of $\mathrm{D}\left(\operatorname{Hom}\left(G, \mathbb{G}_{m}\right)\right)$, by the universal property it follows that $\delta$ is surjective.

Example 1.6. Let $K$ be a field extension of $k$ which is not perfect. We claim that:

(1) there are subgroups $H$ of $\pi^{\mathrm{L}}(K / k)$ which are not of the form $\pi^{\mathrm{L}}(L / k)$ for some purely inseparable extension $L / K$;

(2) if $L / K$ is a nontrivial purely inseparable extension, then the quotient fpqc space $\pi^{\mathrm{L}}(K / k) / \pi^{\mathrm{L}}(L / k)$ is not finite over $k$. 
From 1.3 and 1.5 we obtain a canonical surjective map $\pi^{\mathrm{L}}(K / k) \longrightarrow \mathrm{D}\left(\left(K^{\text {perf }}\right)^{*} / K^{*}\right)$ which is universal among maps to a diagonalizable group scheme. Given a purely inseparable extension $L / K$ we obtain a commutative diagram

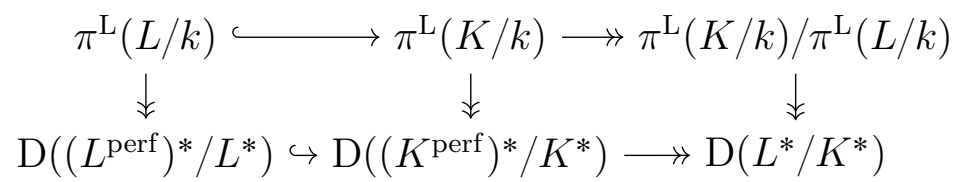

In particular if $K^{*} \subseteq Q \subseteq\left(K^{\text {perf }}\right)^{*}$ is a subgroup not of the form $L^{*}$ for some purely inseparable extension $L$ of $K$ then the inverse image of $\mathrm{D}\left(\left(K^{\text {perf }}\right)^{*} / Q\right) \subseteq \mathrm{D}\left(\left(K^{\text {perf }}\right)^{*} / K^{*}\right)$ along $\pi^{\mathrm{L}}(K / k) \longrightarrow \mathrm{D}\left(\left(K^{\text {perf }}\right)^{*} / K^{*}\right)$ cannot be a local fundamental group. For $(2)$ instead one just has to show that $L^{*} / K^{*}$ is not finitely generated. Indeed one observes that the vertical map on the right is faithfully flat because all other surjective maps are affine and faithfully flat (see [SP21, 036J]).

In order to have a concrete example for (1) and also show (2) it is enough to prove that, if $L=K[X] /\left(X^{p}-\lambda\right)$ with $\lambda \in K-K^{p}$, then $L^{*} / K^{*}$ is not finitely generated. Since $L^{*} / K^{*}$ is an $\mathbb{F}_{p^{-}}$vector space it is enough to show that $L^{*} / K^{*}$ is infinite. Set $v_{n}=1+\lambda^{n} X$ for $n \in \mathbb{N}$. We claim that all these elements are different in $L^{*} / K^{*}$. If $v_{m}=v_{n}$ for some $m \neq n \in \mathbb{N}$ then a direct computation shows that $\lambda$ is a root of unity. In particular it is algebraic and thus separable over $k$. In conclusion $X$ is purely inseparable over the perfect field $k(\lambda)$, from which we find the contradiction $X \in k(\lambda) \subseteq K$.

\section{The local Nori fundamental group in General}

In this section we fix a base field $k$ of positive characteristic $p$.

Definition 2.1. A group scheme $G$ over $k$ is called local if it is finite and connected.

An affine gerbe $\Gamma$ over $k$ is called finite (resp. finite and local) if $\Gamma \times_{k} \bar{k} \simeq \mathrm{B}_{\bar{k}} G$, where $G$ is a finite (resp. finite and local) group scheme over $\bar{k}$.

By a pro-local gerbe (resp. pro-local group scheme) over $k$ we mean a small cofiltered limit of finite and local gerbes (resp. group schemes) over $k$.

Definition 2.2. Let $\mathcal{X}$ be an algebraic stack over $k$. A local Nori fundamental gerbe of $\mathcal{X} / k$ is a pro-local gerbe $\Pi$ over $k$ together with a morphism $\mathcal{X} \longrightarrow \Pi$ such that for all finite and local gerbes $\Gamma$ over $k$ the pullback functor

$$
\operatorname{Hom}_{k}(\Pi, \Gamma) \longrightarrow \operatorname{Hom}_{k}(\mathcal{X}, \Gamma)
$$

is an equivalence. If such a gerbe exists, it is unique and will be denoted by $\Pi_{\mathcal{X} / k}^{\mathrm{L}}$.

Remark 2.3. Notice that a gerbe $\Gamma$ is finite (resp. finite and local) if and only it is finite (resp. finite and local) in the sense of [TZ19, Definition 3.1, p. 10] (resp. [TZ19, Definition 3.9, p. 12]). (See [TZ19, Proposition B.6, p. 41])

By [TZ19, Theorem 7.1], if $\mathcal{X}$ is non-empty, reduced and $\mathrm{H}^{0}\left(\mathcal{O}_{\mathcal{X}}\right)$ does not contain nontrivial purely inseparable field extensions of $k$, then the local Nori fundamental gerbe $\Pi_{\mathcal{X} / k}^{\mathrm{L}}$ exists and coincides with the local Nori fundamental gerbe considered in [TZ19, 
Definition 4.1, p. 12]. Moreover, the local Nori fundamental gerbe is unique up to a unique isomorphism because pro-local gerbes are projective limits of finite and local gerbes.

Theorem 2.4. [TZ19, Theorem 7.1] Assume that $\mathcal{X}$ is reduced and $\mathrm{H}^{0}\left(\mathcal{O}_{\mathcal{X}}\right)$ does not contain nontrivial purely inseparable field extensions of $k$. Denote by $F: \mathcal{X} \rightarrow \mathcal{X}$ and $F_{k}:$ Spec $k \rightarrow$ Spec $k$ the absolute Frobenius morphisms. For $i \in \mathbb{N}$ denote by $\mathcal{D}_{i}$ the category of triples $(\mathcal{F}, V, \lambda)$ where $\mathcal{F} \in \operatorname{Vect}(\mathcal{X}), V \in \operatorname{Vect}(k)$ and $\lambda: F^{i *} \mathcal{F} \rightarrow V \otimes_{k} \mathcal{O}_{\mathcal{X}}$ is an isomorphism. Then the category $\mathcal{D}_{i}$ is $k$-Tannakian with $k$-structure $k \rightarrow \operatorname{End}_{\mathcal{D}_{i}}\left(\mathcal{O}_{\mathcal{X}}, k, \mathrm{id}\right)$, $x \mapsto\left(x, x^{p^{i}}\right)$. Moreover the functor

$$
\mathcal{D}_{i} \longrightarrow \mathcal{D}_{i+1},(\mathcal{F}, V, \lambda) \longmapsto\left(\mathcal{F}, F_{k}^{*} V, F^{*} \lambda\right)
$$

is $k$-linear, monoidal and exact, and there is a natural equivalence of $k$-Tannakian categories:

$$
\mathcal{D}_{\infty}:=\lim _{i \in \mathbb{N}} \mathcal{D}_{i} \stackrel{\simeq}{\longrightarrow} \operatorname{Rep}\left(\Pi_{\mathcal{X} / k}^{\mathrm{L}}\right) .
$$

Proposition 2.5. With notation from 2.4 the functors

$$
\mathcal{D}_{i} \rightarrow \mathcal{D}_{i+1}
$$

and therefore the functors $\mathcal{D}_{i} \rightarrow \mathcal{D}_{\infty}$ are fully faithful. In particular $\mathcal{D}_{i}$ is a sub-Tannakian category of $\mathcal{D}_{j}$ for $j>i$ and $j=\infty$.

Proof. The last claim is a consequence of [TZ19, Remark B.7]: the full faithfulness is enough to conclude that the corresponding map on gerbes is a quotient.

Consider $E=(\mathcal{E}, V, \lambda), E^{\prime}=\left(\mathcal{E}^{\prime}, V^{\prime}, \lambda^{\prime}\right) \in \mathcal{D}_{i}$. We have

$$
\operatorname{Hom}\left(E, E^{\prime}\right)=\left\{(\alpha, \beta) \in \operatorname{Hom}\left(\mathcal{E}, \mathcal{E}^{\prime}\right) \times \operatorname{Hom}\left(V, V^{\prime}\right) \mid \lambda^{\prime} F^{i *}(\alpha)=\left(\beta \otimes \mathcal{O}_{\mathcal{X}}\right) \lambda\right\} .
$$

The functor $\Phi: \mathcal{D}_{i} \rightarrow \mathcal{D}_{i+1} \operatorname{maps}(\mathcal{E}, V, \lambda)$ to $\left(\mathcal{E}, F_{k}^{*} V, F_{k}^{*} \lambda\right)$ and $(\alpha, \beta) \in \operatorname{Hom}\left(E, E^{\prime}\right)$ to $\left(\alpha, F_{k}^{*} \beta\right)$. Let $(\alpha, \delta) \in \operatorname{Hom}\left(\Phi(E), \Phi\left(E^{\prime}\right)\right)$. Fix isomorphisms $V \simeq k^{n}$ and $V^{\prime} \simeq k^{m}$, so that $F_{k}^{*} V \simeq k^{n}$ and $F_{k}^{*} V^{\prime} \simeq k^{m}$. The map $\delta: F_{k}^{*} V \rightarrow F_{k}^{*} V^{\prime}$ is therefore a matrix $\delta=\left(\delta_{i j}\right)$ with $\delta_{i j} \in k$. Consider the map

$$
\beta=\lambda^{\prime} F^{i *}(\alpha) \lambda^{-1}: V \otimes \mathcal{O}_{\mathcal{X}} \rightarrow V^{\prime} \otimes \mathcal{O}_{\mathcal{X}}
$$

This is represented by a matrix $\beta=\left(\beta_{i j}\right)$ with $\beta_{i j} \in \mathrm{H}^{0}\left(\mathcal{O}_{\mathcal{X}}\right)$. The hypothesis is that $\beta_{i j}^{p}=\delta_{i j}$. Since $\mathcal{X}$ is reduced, we have $\beta_{i j} \in k$. Thus $(\alpha, \beta) \in \operatorname{Hom}\left(E, E^{\prime}\right)$ induces $(\alpha, \delta)$.

We now consider the case of a perfect field. In this case the local Nori gerbe exists for all reduced algebraic stacks.

Remark 2.6. Using the same notations from 2.4 and assuming that $k$ is perfect, the functors $\mathcal{D}_{n} \longrightarrow \operatorname{Vect}(k),(\mathcal{F}, V, \lambda) \longmapsto F_{k}^{-n^{*}} V$, where $F_{k}$ is the absolute Frobenius of $k$, are compatible when $n$ varies, so they induce a functor $\mathcal{D}_{\infty} \longrightarrow \operatorname{Vect}(k)$. It is easy to check that this functor is $k$-linear, exact and tensorial. In particular $\mathcal{D}_{\infty}$ has a neutralization or, in other words $\Pi_{\mathcal{X} / k}^{\mathrm{L}}(k) \neq \varnothing$. 
In fact there is much more: over a perfect field a pro-local gerbe is neutral, and the neutralization is unique up to a unique isomorphism.

Lemma 2.7. Let $\Gamma$ be a pro-local gerbe over a perfect field $k$. Then $\Gamma(k)$ is equivalent to a set with one point, in other words, it is a non-empty groupoid in which between every two objects there exists exactly one isomorphism. Equivalently, the Tannakian category $\operatorname{Vect}(\Gamma)$ has a neutral fiber functor which is unique up to a unique isomorphism.

Proof. Since $\Gamma$ is a profinite gerbe, we may write $\Gamma:=\lim _{i \in I} \Gamma_{i}$, where $I$ is a cofiltered essentially small category and $\Gamma_{i}$ are finite and local gerbes over $k$. In this way we reduce to the case where $\Gamma$ is finite.

We first show that $\Gamma(k) \neq \varnothing$. The stack $\Gamma$ is reduced because it has a faithfully flat map from a reduced scheme, namely the spectrum of some field. Moreover since $\Gamma$ is local we clearly have $\Gamma=\Pi_{\Gamma / k}^{\mathrm{L}}$. Thus $\Gamma(k)=\Pi_{\mathcal{X} / k}^{\mathrm{L}}(k) \neq \varnothing$ by 2.6 .

In particular $\Gamma=\mathrm{B} G$, where $G$ is a finite and local group scheme over $k$. If $P$ is a $G$-torsor over $k$ then $P$ is finite and geometrically connected. Thus $P=\operatorname{Spec} A$, where $A$ is local, finite with purely inseparable residue field extension $l / k$. Since $k$ is perfect, we have $l=k$. Thus $P(k)$ consists of one element, as we wanted to show.

Remark 2.8. The key point of Lemma 2.7 is that any non-empty finite and local stack over a perfect field $k$ has a $k$-rational point. Indeed, consider the $n$-th relative Frobenius twist: $\Gamma \longrightarrow \Gamma^{(n)}$. According to [TZ19, Lemma 3.6, p. 11] there exists $n \in \mathbb{N}$ such that the Frobenius twist factors through $\Gamma_{\text {ét }}$ which is equal to $\operatorname{Spec}(k)$ because $\Gamma$ is finite and local. This provides a rational section for $\Gamma^{(n)}$. But since $k$ is perfect, by twisting back with $(-)^{(-n)}$, we see that $\Gamma$ has a $k$-rational point.

Since affine group schemes are the same as affine gerbes with a given rational section, we obtain the following:

Corollary 2.9. Let $k$ be a perfect field. The functor

$$
\begin{aligned}
\text { \{pro-local group schemes over } k\} & \longrightarrow\{\text { pro-local gerbes over } k\} \\
G & \longmapsto \mathrm{B} G
\end{aligned}
$$

is an equivalence of categories (meaning that between two functors of pro-local gerbes there exists at most one natural isomorphism).

Definition 2.10. Let $\mathcal{X}$ be a reduced algebraic stack over a perfect field $k$. We denote by $\pi^{\mathrm{L}}(\mathcal{X} / k)$ the pro-local group scheme such that $\Pi_{\mathcal{X} / k}^{\mathrm{L}}=\mathrm{B} \pi^{\mathrm{L}}(\mathcal{X} / k)$ and call it the local Nori fundamental group scheme of $\mathcal{X}$ over $k$.

Definition 2.11. If $G$ is an affine group scheme over $k$, a $G$-torsor $P \rightarrow X$ is minimal if it is not induced by a torsor under a strict subgroup of $G$.

Remark 2.12. This notion of minimality for a torsor is similar but different from the Nori reduced one (see [BV15, Definition 5.10]). Indeed for a $G$-torsor $P \rightarrow X$ being Nori reduced means that for any factorization

$$
X \rightarrow \Gamma \stackrel{\gamma}{\longrightarrow} \mathrm{B} G
$$


where $\Gamma$ is a finite gerbe and $\gamma$ is faithful, the map $\gamma$ is an isomorphism. Minimality requires the same property, but under the additional assumption that $\Gamma \simeq \mathrm{B} H$ and $\gamma$ "preserves the trivial torsors", that is, it is induced by a homomorphism $H \rightarrow G$.

We see therefore that Nori reduced implies minimal, but the converse is not true in general: if $L / k$ is a Galois extension with group $G$ then $\operatorname{Spec} L \rightarrow \operatorname{Spec} k$ is a minimal $G$-torsor while the corresponding map Spec $k \rightarrow \mathrm{B} G$ is not Nori reduced.

On the other hand the two notions coincide in the following two cases.

- The field $k$ is algebraically closed, because in this case for any finite gerbe $\Gamma$ the category $\Gamma(k)$ is non-empty, so that $\Gamma \simeq \mathrm{B} H$ is neutral, and any $H$-torsor over $k$ is trivial.

- The group $G$ is a pro-local group scheme, thanks to Corollary 2.9.

Proposition 2.13. Let $\mathcal{X}$ be a reduced algebraic stack over a perfect field $k$. Then $\pi^{\mathrm{L}}(\mathcal{X} / k)$ is the unique pro-local fundamental group scheme over $k$ with natural equivalences

$$
\operatorname{Hom}_{k}\left(\pi^{\mathrm{L}}(\mathcal{X} / k), G\right) \longrightarrow\{G \text {-torsors over } \mathcal{X}\}
$$

functorial in the finite and local group scheme $G$ over $k$. Moreover a group homomorphism

$$
\pi^{\mathrm{L}}(\mathcal{X} / k) \longrightarrow G
$$

is surjective if and only if the corresponding G-torsor is minimal.

Proof. This follows from the universal property of the local Nori gerbe and the equivalence in 2.9. In particular the category on the right is indeed a set, that is there exists at most one isomorphism between two $G$-torsors over $\mathcal{X}$.

Remark 2.14. An alternative way to state 2.13 is that there are natural bijections

$$
\operatorname{Hom}_{k}\left(\pi^{\mathrm{L}}(\mathcal{X} / k), G\right) \simeq \mathrm{H}^{1}(\mathcal{X}, G)
$$

functorial in the finite, local group scheme $G$ over $k$.

In this special situation of local group schemes there is no need for choosing a rational or geometric point. A similar phenomenon also appears in [Zh18, Proposition 2.21 (ii) and Remark 2.22]. The following proposition shows that this is indeed not a coincidence:

Proposition 2.15. Let $\mathcal{X}$ be a reduced algebraic stack over a perfect field $k$ and consider the category $\mathcal{N}(\mathcal{X} / k)$ of pairs $(G, \mathcal{P})$ where $G$ is a finite and local group scheme over $k$ and $f: \mathcal{P} \longrightarrow \mathcal{X}$ is a $G$-torsor. Then $\mathcal{N}(\mathcal{X} / k)$ is a small cofiltered category and there is a canonical isomorphism

$$
\pi^{\mathrm{L}}(\mathcal{X} / k) \simeq \lim _{(G, \mathcal{P}) \in \mathcal{N}(\mathcal{X} / k)} G
$$

Proof. By the above discussion we obtain that the category $\mathcal{N}(\mathcal{X} / k)$ is equivalent to the category $\operatorname{Hom}_{k}\left(\pi^{\mathrm{L}}(\mathcal{X} / k),-\right)$ of morphisms from $\pi^{\mathrm{L}}(\mathcal{X} / k)$ to finite and local group schemes. Notice that $\operatorname{Hom}_{k}\left(\pi^{\mathrm{L}}(\mathcal{X} / k),-\right)$ has fiber products and, in particular, it is cofiltered (see 
[Zh18, Remark 1.3, (i)]). Moreover, if $\mathcal{N}^{\prime}(\mathcal{X} / k)$ is the full subcategory of $\operatorname{Hom}_{k}\left(\pi^{\mathrm{L}}(\mathcal{X} / k),-\right)$ consisting of quotient maps, then we have an isomorphism

$$
\lim _{\mathcal{N}(\mathcal{X} / k)} G \stackrel{\simeq}{\longrightarrow} \lim _{\mathcal{N}^{\prime}(\mathcal{X} / k)} G
$$

which is the limit of all finite and local quotients of $\pi^{\mathrm{L}}(\mathcal{X} / k)$. Thus the Hopf algebra of $\lim _{\mathcal{N}(\mathcal{X} / k)} G$ is contained in $k\left[\pi^{\mathrm{L}}(\mathcal{X} / k)\right]$. Since $\pi^{\mathrm{L}}(\mathcal{X} / k)$ is pro-local, it is a cofiltered limit of some of its finite and local quotients. In this way we obtain an inclusion of Hopf algebras in the other direction, and this finishes the proof.

Corollary 2.16. If $X$ is a reduced scheme over a perfect field $k$ with a geometric point $x: \operatorname{Spec} \Omega \longrightarrow X$, where $\Omega$ is an algebraically closed field, then $\pi^{\mathrm{L}}(X / k)$ coincides with the group scheme $\pi^{\mathrm{L}}(X / k, x)$ defined in [Zh18, Definition 4.5(iv)].

Proof. The right-hand side of the isomorphism in 2.15 is the group scheme $\pi^{\mathrm{L}}(X / k, x)$ as defined in [Zh18, Definitions 3.6 and 4.5(iv)].

Proposition 2.17. If $K / k$ is a field extension with $k$ perfect, then we have an isomorphism between $\pi^{\mathrm{L}}(K / k)$ as defined in 1.1 and $\pi^{\mathrm{L}}(\operatorname{Spec} K / k)$ as defined in 2.10.

Proof. Using notations from 2.4 for $\mathcal{X}=\operatorname{Spec} K$ we have to show that there is an equivalence of Tannakian categories $\mathcal{D}_{\infty} \simeq \mathcal{D}_{\infty}(K / k)$. If $(V, W, \phi) \in \mathcal{D}_{n}(K / k)$ with $V \in \operatorname{Vect}(K)$, $W \in \operatorname{Vect}(k)$ and $\phi: F^{n^{*}} V \simeq W \otimes_{k} K$, then via the isomorphism of fields $K^{1 / p^{n}} \rightarrow K$, $x \mapsto x^{p^{n}}$, we get $F^{n^{*}} V \simeq V \otimes_{K} K^{1 / p^{n}}$ and $W \otimes_{k} K \simeq\left(F_{k}^{-n^{*}} W\right) \otimes_{k} K^{1 / p^{n}}$. Then it is not difficult to show that, for $n \in \mathbb{N}$, the category $\mathcal{D}_{n}(K / k)$ is equivalent to the category of triples $(M, N, \varphi)$ where $M \in \operatorname{Vect}(K), N \in \operatorname{Vect}(k)$ and $\varphi: M \otimes_{K} K^{1 / p^{n}} \simeq N \otimes_{k} K^{1 / p^{n}}$ is an isomorphism. By passing to the limit we get $\mathcal{D}_{\infty} \simeq \mathcal{D}_{\infty}(K / k)$.

We conclude this section by showing that the local fundamental group detects isomorphisms. We need the following lemma.

Lemma 2.18. Let $K / k$ be an extension of fields. Then there are natural isomorphisms:

$$
\begin{array}{ll}
\operatorname{Hom}\left(\pi^{\mathrm{L}}(K / k), \alpha_{p^{n}}\right) \simeq K / K^{p^{n}} & \text { and } \operatorname{Hom}\left(\pi^{\mathrm{L}}(K / k), \mathbb{G}_{a}\right) \simeq K^{\text {perf }} / K, \\
\operatorname{Hom}\left(\pi^{\mathrm{L}}(K / k), \mu_{p^{n}}\right) \simeq K^{*} / K^{* p^{n}} \quad \text { and } \operatorname{Hom}\left(\pi^{\mathrm{L}}(K / k), \mathbb{G}_{m}\right) \simeq\left(K^{\text {perf }}\right)^{*} / K^{*} .
\end{array}
$$

Proof. The isomorphism for $\alpha_{p^{n}}$ follows from 2.14. Moreover, the isomorphisms for $n$ and for $n+1$ fit in a commutative diagram:

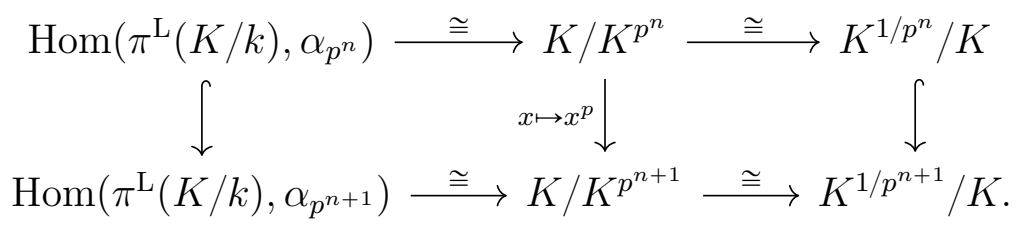

Since $\pi^{\mathrm{L}}(K / k)$ is pro-local, we have:

$$
\operatorname{Hom}\left(\pi^{\mathrm{L}}(K / k), \mathbb{G}_{a}\right) \simeq \underset{n}{\lim } \operatorname{Hom}\left(\pi^{\mathrm{L}}(K / k), \alpha_{p^{n}}\right) .
$$


The transition maps are described in the above commutative diagram, hence

$$
\operatorname{Hom}\left(\pi^{\mathrm{L}}(K / k), \mathbb{G}_{a}\right) \simeq \underset{n}{\lim _{\longrightarrow}} K^{1 / p^{n}} / K=K^{\text {perf }} / K .
$$

In a similar way, one retrieves the isomorphism for $\mu_{p^{n}}$ from 2.14 and derives the isomorphism for $\mathbb{G}_{m}$ (which was alternatively obtained in 1.3).

Proposition 2.19. Let $k$ be a perfect field and $E / K$ be a finitely generated extension of fields over $k$. Then the map

$$
\pi^{\mathrm{L}}(E / k) \rightarrow \pi^{\mathrm{L}}(K / k)
$$

is an isomorphism if and only if $K=E$ or $E / K$ is a finite extension of perfect fields.

Proof. The if part follows from 1.4. Assume that the map on fundamental groups is an isomorphism. By 2.18 the map

$$
K^{\text {perf }} / K \rightarrow E^{\text {perf }} / E
$$

is an isomorphism. In particular the map $K^{\text {perf }} \rightarrow E^{\text {perf }} / E$ is surjective, and $E^{\text {perf }}=$ $K^{\text {perf }}(E)$. Write $K \subseteq F \subseteq E$ with $F / K$ purely transcendental and $E / F$ finite. We have

$$
K^{\text {perf }} \subseteq K^{\text {perf }}(F) \subseteq K^{\text {perf }}(E)=E^{\text {perf }} .
$$

Since the finitely generated extension $K^{\text {perf }}(E)=E^{\text {perf }}$ over $K^{\text {perf }}$ contains the extension $K^{\text {perf }}(F) / K^{\text {perf }}, K^{\text {perf }}(F)$ cannot contain indeterminates, that is $F=K$ and $E / K$ is finite.

Now split $E / K$ as $K \subseteq S \subseteq E$ with $S / K$ separable and $E / S$ purely inseparable. Let $n$ be an index such that $E^{p^{n}} \subseteq S$. By 2.18 the composition

$$
K / K^{p^{n}} \rightarrow S / S^{p^{n}} \rightarrow E / E^{p^{n}}
$$

is an isomorphism. In particular the second map is surjective. But, since $E^{p^{n}} \subseteq S$, the image of the second map is $S / E^{p^{n}}$ and therefore $S=E$, that is $E / K$ is separable. that

So $E=K[x] /(f(x))$, for some separable polynomial $f \in K[x]$. The separability implies

$$
E^{\text {perf }}=K^{\text {perf }}[x] /(f(x))
$$

so that the isomorphism (1) is the inclusion

$$
K^{\text {perf }} / K \rightarrow\left(K^{\text {perf }} / K\right)^{\operatorname{deg} f} .
$$

It follows that either $K=K^{\text {perf }}$, so that also $E$ is perfect, or $\operatorname{deg} f=1$, that is $K=E$.

\section{Generic surjectivity}

We fix a perfect field $k$ of characteristic $p>0$. The aim of this section is to show Theorem II and related results.

Let $\mathcal{X}$ be an algebraic stack over $k$, and let $G$ be a finite local $k$-group scheme. Firstly, we look for a criterion for the surjectivity of the map induced on local fundamental group schemes. 
Remark 3.1. If $\mathcal{P} \longrightarrow \mathcal{X}$ is a $G$-torsor over an algebraic stack $\mathcal{X}$ and $H \subseteq G$ is a subgroup then $\mathcal{P}$ is induced by an $H$-torsor if and only if $\mathcal{P} / H \longrightarrow \mathcal{X}$ has a section.

Indeed, if $\mathcal{P} / H \longrightarrow \mathcal{X}$ admits a section, then the $H$-torsor inducing $\mathcal{P} \longrightarrow \mathcal{X}$ is just the pullback of $\mathcal{P} \longrightarrow \mathcal{P} / H$ along the section. Conversely, if $\mathcal{P} \longrightarrow \mathcal{X}$ reduces to a $H$-torsor $\mathcal{Q} \longrightarrow \mathcal{X}$, then the map

$$
\mathcal{X} \simeq \mathcal{Q} / H \longrightarrow \mathcal{P} / H \longrightarrow \mathcal{X}
$$

provides a section.

Remark 3.2. If $\mathcal{X}$ is a reduced algebraic stack over $k, \mathcal{P} \rightarrow \mathcal{X}$ is a torsor under a finite local group scheme $G$ over $k$ and $H \subseteq G$ is a subgroup then $\mathcal{P} / H \rightarrow \mathcal{X}$ is a finite universal homeomorphism. In particular $\mathcal{P} \rightarrow \mathcal{X}$ is induced by an $H$-torsor if and only if $(\mathcal{P} / H)_{\text {red }} \rightarrow \mathcal{X}$ is an isomorphism.

Remark 3.3. If $\mathcal{V} \longrightarrow \mathcal{X}$ is a map between reduced algebraic stacks then $\pi^{\mathrm{L}}(\mathcal{V} / k) \longrightarrow$ $\pi^{\mathrm{L}}(\mathcal{X} / k)$ is surjective if and only if the following condition holds: if $\mathcal{P} \longrightarrow \mathcal{X}$ is a minimal $G$-torsor for a local group scheme $G$ then $\mathcal{P} \times \mathcal{X} \mathcal{V} \longrightarrow \mathcal{V}$ is also minimal.

Indeed, the condition that the pullback of any minimal finite local $G$-torsor on $\mathcal{X}$ to $\mathcal{V}$ is minimal is equivalent to the condition that any surjective map $\pi^{\mathrm{L}}(\mathcal{X} / k) \longrightarrow G$ is still surjective after composing with $\pi^{\mathrm{L}}(\mathcal{V} / k) \longrightarrow \pi^{\mathrm{L}}(\mathcal{X} / k)$, and this is equivalent to saying that the map $\pi^{\mathrm{L}}(\mathcal{V} / k) \longrightarrow \pi^{\mathrm{L}}(\mathcal{X} / k)$ itself is surjective.

Putting together 3.1 and 3.3 we obtain the following criterion.

Lemma 3.4. Let $\mathcal{V} \longrightarrow \mathcal{X}$ be a map between reduced algebraic stacks. Assume that if $\mathcal{P} \rightarrow \mathcal{X}$ is a minimal torsor under a finite local group scheme $G$ over $k$ and $H \subseteq G$ is a subgroup then any map $\mathcal{V} \rightarrow \mathcal{P} / H$ over $\mathcal{X}$ extends to a section of $\mathcal{P} / H \rightarrow \mathcal{X}$. Then

$$
\pi^{\mathrm{L}}(\mathcal{V} / k) \rightarrow \pi^{\mathrm{L}}(\mathcal{X} / k)
$$

is surjective.

Lemma 3.5. Let $K$ be a field extension of $k$. Let $E$ be a non-zero $K$-algebra such that if $e \in E$ and $e^{p} \in K$ then $e \in K$ (e.g. when $E$ is geometrically reduced over $K$ ). Then the map

$$
\pi^{\mathrm{L}}(E / k) \rightarrow \pi^{\mathrm{L}}(K / k)
$$

is surjective.

Proof. The condition on $E$ implies that $E$ is reduced, so that $\pi^{\mathrm{L}}(E / k)$ exists by 2.3 .

Following 3.4 let $P \rightarrow \operatorname{Spec} K$ be a minimal $G$-torsor with a map Spec $E \rightarrow P / H$ over $K$. By 3.2 we see that $(P / H)_{\text {red }} \rightarrow \operatorname{Spec} K$ is a finite universal homeomorphism, that is $(P / H)_{\text {red }}=\operatorname{Spec} F$ for a finite purely inseparable field extension $F / K$, and we need to show that it is an isomorphism.

Since $E$ is reduced we have factorizations $\operatorname{Spec} E \rightarrow(P / H)_{\text {red }} \subseteq P / H$ and therefore

$$
K \subseteq F \subseteq E
$$

The condition on $E$ implies $F=K$ as required. 
Corollary 3.6. The map

$$
\pi^{\mathrm{L}}(k((t)) / k) \rightarrow \pi^{\mathrm{L}}(k(t) / k)
$$

is surjective.

Proof. From 3.5 we just have to show that if $u \in k((t))$ and $u^{p} \in k(t)$, then $u \in k(t)$. Multiplying $u$ with a denominator of $u^{p}$ we may assume that $u^{p} \in k[t]$. Now $u=\sum u_{i} t^{i}$ is a Laurent series, and $u_{i}^{p}=0$ for $i \gg 0$, hence $u_{i}=0$ for $i \gg 0$, hence $u$ is a rational function.

Proposition 3.7. Let $E / k$ be a finitely generated extension of transcendence degree $n$. Then there are elements $t_{1}, \ldots, t_{n} \in E$ algebraically independent over $k$ such that

$$
\pi^{\mathrm{L}}(E / k) \longrightarrow \pi^{\mathrm{L}}\left(k\left(t_{1}, \ldots, t_{n}\right) / k\right)
$$

is surjective. In particular, if $E / k$ is not finite, there exists an indeterminate $t \in E$ and a surjective map

$$
\pi^{\mathrm{L}}(E / k) \rightarrow \pi^{\mathrm{L}}(k(t) / k)
$$

Proof. By [SP21, 030Q] there is a subfield $k \subseteq F \subseteq E$ such that $F / k$ is purely transcental of degree $n$ and $E / F$ is finite and separable. We see that both extensions $E / F$ and $F / k\left(t_{1}\right)$ satisfy the hypothesis of 3.5 , proving the result.

Lemma 3.8. Let $f: \mathcal{Y} \longrightarrow \mathcal{X}$ be a faithfully flat geometrically reduced map of reduced algebraic stacks over $k$. Then the induced map

$$
\pi^{\mathrm{L}}(\mathcal{Y} / k) \rightarrow \pi^{\mathrm{L}}(\mathcal{X} / k)
$$

is surjective.

Proof. Following 3.4 let $\mathcal{P} \rightarrow \mathcal{X}$ be a minimal $G$-torsor with a map $\mathcal{Y} \rightarrow \mathcal{P} / H$ over $\mathcal{X}$.

Since $f$ is geometrically reduced and thanks to 3.2 we have that

$$
((\mathcal{P} / H) \times \mathcal{X} \mathcal{Y})_{\text {red }}=(\mathcal{P} / H)_{\text {red }} \times \mathcal{X} \mathcal{Y} \rightarrow \mathcal{Y}
$$

is an isomorphism. In other words the map $(\mathcal{P} / H)_{\text {red }} \rightarrow \mathcal{X}$ is an isomorphism after pulling back to $\mathcal{Y}$ and, again by 3.2 , we need to show that is an isomorphism.

For this, we can replace $\mathcal{X}$ by an affine open of an atlas and, since $(\mathcal{P} / H)_{\text {red }} \longrightarrow \mathcal{X}$ is affine, also assume it is the spectrum of a local ring. In this case $\mathcal{Y} \longrightarrow \mathcal{X}$ is automatically an fpqc covering, and by descent we get the result.

Lemma 3.9. Let $\mathcal{X}$ be a reduced algebraic stack over $k$. Then

$$
\pi^{\mathrm{L}}(\mathcal{X} / k) \rightarrow \pi^{\mathrm{L}}\left(\mathrm{H}^{0}\left(\mathcal{O}_{\mathcal{X}}\right) / k\right)
$$

is surjective.

Proof. Following 3.4 let $P \longrightarrow$ Spec $\mathrm{H}^{0}\left(\mathcal{O}_{\mathcal{X}}\right)$ be a minimal $G$-torsor with a map $\mathcal{X} \rightarrow P / H$ over $\mathrm{H}^{0}\left(\mathcal{O}_{\mathcal{X}}\right)$. The desired factorization exists because $P / H$ is affine. 
In the following, we will deal with normal algebraic stacks. These are algebraic stacks which admit a smooth atlas from a normal scheme in the sense of [SP21, 033H]. In particular, these algebraic stacks may not be locally Noetherian. Notice also that any smooth atlas of a normal algebraic stack is normal (see [SP21, 04YH]).

We need a technical lemma.

Lemma 3.10. Let $\mathcal{X}$ be a quasi-separated algebraic stack and $f: V \rightarrow \mathcal{X}$ be an open and locally of finite type map from a scheme. Then:

(1) the morphism $f$ maps generic points into generic points;

(2) if $\mathcal{X}$ is irreducible then all generic points of $V$ have an open irreducible neighborhood;

(3) if $V$ is quasi-compact and $\mathcal{Z}$ is an irreducible component of $\mathcal{X}$ then $f^{-1}(\mathcal{Z})$ is either empty or a finite union of irreducible components $W$ of $V$ such that $W \rightarrow \mathcal{Z}$ is dominant.

Proof. Notice that the topological space $|\mathcal{X}|$ is sober by [SP21, 0DQQ].

$(3) \Longrightarrow(1)$. Let $v \in V$ be a generic point and $\xi \in|\mathcal{X}|$ a generic point such that $f(v) \in \overline{\{\xi\}}$. Replacing $V$ by a quasi-compact open neighborhood of $v$ and applying (3), we can conclude that the map $\overline{\{v\}} \rightarrow \overline{\{\xi\}}$ is dominant, which means $f(v)=\xi$

$(3) \Longrightarrow(2)$. Let $v \in V$ be a generic point and $U \subseteq V$ a quasi-compact open subset such that $v \in U$. By (3) applied to $\mathcal{Z}=\mathcal{X}$, it follows that $U$ has finitely many irreducible components. Thus it is enough to remove from $U$ all the irreducible components not containing $v$.

(3). As $\mathcal{X}$ is quasi-separated and $V$ is quasi-compact it follows that $f: V \rightarrow \mathcal{X}$ is a quasi-compact map. We can assume $f^{-1}(\mathcal{Z}) \neq \varnothing$. Let $\xi$ be the generic point of $\mathcal{Z}$ and set $T=f^{-1}(\xi)$.

We have $\bar{T}=f^{-1}(\mathcal{Z})$, so that, in particular, $T \neq \varnothing$. Indeed otherwise we would have the contradiction

$$
f(V-\bar{T}) \cap \mathcal{Z} \neq \varnothing \Longrightarrow \xi \in f(V-\bar{T}) \Longrightarrow T \cap(V-\bar{T}) \neq \varnothing
$$

Here we used that $f(V-\bar{T})$ is open as $f$ is open.

If $\operatorname{Spec} \Omega \rightarrow \mathcal{X}$ is a geometric point mapping to $\xi$, the continuous map $V_{\Omega}=\operatorname{Spec} \Omega \times \mathcal{X}$ $V \rightarrow V$ surjects onto $T$. As $f$ is quasi-compact and locally of finite type, it follows that $V_{\Omega}$ is of finite type over $\Omega$. If $t_{1}, \ldots, t_{r}$ are the image of the (finitely many) generic points of $V_{\Omega}$ along the map $V_{\Omega} \rightarrow T$, then

$$
T=\overline{\left\{t_{1}\right\}} \cup \cdots \cup \overline{\left\{t_{r}\right\}}
$$

where the closure is taken inside the topological space $T$. Taking the closure now inside $V$ we can conclude that the generic points of $\bar{T}=f^{-1}(\mathcal{Z})$ are among the $t_{1}, \ldots, t_{r}$. So they are finitely many and they all maps to the generic point $\xi$ as required.

Lemma 3.11. Let $\mathcal{X}$ be a normal, quasi-separated and irreducible algebraic stack over $k$, and let $\mathcal{U} \subseteq \mathcal{X}$ be a non-empty open substack. Then the map

$$
\pi^{\mathrm{L}}(\mathcal{U} / k) \longrightarrow \pi^{\mathrm{L}}(\mathcal{X} / k)
$$

is surjective. 
Proof. Following 3.4 let $\mathcal{P} \rightarrow \mathcal{X}$ be a minimal $G$-torsor with a section $\mathcal{U} \rightarrow \mathcal{P} / H$ over $\mathcal{X}$. By 3.2 the morphism $(\mathcal{P} / H)_{\text {red }} \rightarrow \mathcal{X}$ is an isomorphism over $\mathcal{U}$ and we need to show that $(\mathcal{P} / H)_{\text {red }} \rightarrow \mathcal{X}$ is an isomorphism as well.

We show that a finite birational map $\mathcal{Y} \rightarrow \mathcal{X}$ from a reduced algebraic stack is an isomorphism. We can assume that $\mathcal{X}$ is quasi-compact and consider a smooth atlas $V \rightarrow \mathcal{X}$ from a quasi-compact scheme.

By 3.10, (3) the scheme $V$ has finitely many irreducible components. Since $V$ is normal, it follows that it is a finite disjoint union of integral normal schemes. Thus we can assume that $\mathcal{X}$ is an integral normal scheme and also that it is affine. Thus $\mathcal{X}=\operatorname{Spec} D$, for a normal domain $D$ and $\mathcal{Y}=\operatorname{Spec} B$, for a reduced ring $B$.

Since $\mathcal{Y}$ contains a dense open subset isomorphic to an open subset of $\mathcal{X}$ and therefore irreducible, it follows that $\mathcal{Y}$ is integral. More precisely that $B$ is a domain with the same fraction field of $D$. As $D \rightarrow B$ is an (injective) integral extension and $D$ is normal we can conclude that $D=B$.

Lemma 3.12. Let $X$ be an integral normal scheme over $k$, and let Spec $\Omega \longrightarrow X$ be the generic point of $X$. Then the map

$$
\pi^{\mathrm{L}}(\operatorname{Spec} \Omega / k) \longrightarrow \pi^{\mathrm{L}}(X / k)
$$

is surjective.

Proof. Following 3.4 let $P \rightarrow X$ be a minimal $G$-torsor with a map Spec $\Omega \rightarrow P / H$ over $X$. By 3.2 it follows that $(P / H)_{\text {red }} \rightarrow X$ is a finite universal homeomorphism which is generically an isomorphism. It is therefore an isomorphism: one first reduce to the affine case and then argue as in the end of the proof of 3.11.

Lemma 3.13. Let $\mathcal{X}$ be a normal, quasi-separated, irreducible algebraic stack over $k$. Let $\operatorname{Spec} \Omega$ be a generic point of a smooth atlas of $\mathcal{X}$, then the map

$$
\pi^{\mathrm{L}}(\operatorname{Spec} \Omega / k) \longrightarrow \pi^{\mathrm{L}}(\mathcal{X} / k)
$$

is surjective.

Proof. Let $f: V \longrightarrow \mathcal{X}$ be the smooth atlas. By 3.10, (2) there is an open and irreducible subset $U$ of $V$ containing the given generic point. In particular $U$ is integral and normal. Let $\mathcal{U}=f(U) \subseteq \mathcal{X}$. Then we can decompose $\pi^{\mathrm{L}}(\operatorname{Spec} \Omega / k) \longrightarrow \pi^{\mathrm{L}}(\mathcal{X} / k)$ into

$$
\pi^{\mathrm{L}}(\operatorname{Spec} \Omega / k) \stackrel{\lambda_{1}}{\longrightarrow} \pi^{\mathrm{L}}(U / k) \stackrel{\lambda_{2}}{\longrightarrow} \pi^{\mathrm{L}}(\mathcal{U} / k) \stackrel{\lambda_{3}}{\longrightarrow} \pi^{\mathrm{L}}(\mathcal{X} / k)
$$

where $\lambda_{1}$ is surjective because of $3.12, \lambda_{2}$ is surjective by 3.8 because $U \rightarrow \mathcal{U}$ is geometrically reduced and faithfully flat, and $\lambda_{3}$ is surjective by 3.11, whence the result.

Theorem 3.14. Let $\mathcal{X}$ be a normal, quasi-separated and irreducible algebraic stack over $k, \mathcal{V}$ a reduced algebraic stack and $\mathcal{V} \rightarrow \mathcal{X}$ be a map. Assume that there is a commutative diagram

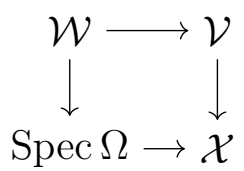


where $\operatorname{Spec} \Omega$ is a generic point of a smooth atlas of $\mathcal{X}$ and $\mathcal{W}$ is a (non empty) reduced algebraic stack with the following property: if $z \in \mathrm{H}^{0}\left(\mathcal{O}_{\mathcal{W}}\right)$ and $z^{p} \in \Omega$ then $z \in \Omega$. Then the map

$$
\pi^{\mathrm{L}}(\mathcal{V} / k) \rightarrow \pi^{\mathrm{L}}(\mathcal{X} / k)
$$

is surjective.

Proof. The map $\pi^{\mathrm{L}}(\operatorname{Spec} \Omega / k) \longrightarrow \pi^{\mathrm{L}}(\mathcal{X} / k)$ is surjective by 3.13. By functoriality it is enough to show that $\pi^{\mathrm{L}}(\mathcal{W} / k) \rightarrow \pi^{\mathrm{L}}(\operatorname{Spec} \Omega / k)$ is surjective. This map factors as

$$
\pi^{\mathrm{L}}(\mathcal{W} / k) \stackrel{\psi}{\rightarrow} \pi^{\mathrm{L}}\left(\operatorname{Spec} \mathrm{H}^{0}\left(\mathcal{O}_{\mathcal{W}}\right) / k\right) \stackrel{\phi}{\rightarrow} \pi^{\mathrm{L}}(\operatorname{Spec} \Omega / k)
$$

Since $\phi$ is surjective by 3.5, while $\psi$ is surjective by 3.9, we get the result.

Remark 3.15. Theorem 3.14 continues to be true if $\mathcal{X}=X$ is a normal integral scheme and $\operatorname{Spec} \Omega \rightarrow X$ is its generic point. Just use 3.12 instead of 3.13.

Proof of Theorem II. Taking into account 3.10, (1), everything follows from 3.14.

\section{REFERENCES}

[Ba18] G. Battiston, A theory of Galois descent for finite inseparable extensions, Proc. Amer. Math. Soc. 146 (2018), no. 1, 69-83.

[BV15] N. Borne, A. Vistoli, The Nori fundamental gerbe of a fibered category, J. Algebraic Geom. 24 (2015), no. 2, 311-353.

[BW20] L. Brantner, J. Waldron, Purely Inseparable Galois theory I: The Fundamental Theorem, (2020) available at https://arxiv.org/abs/2010.15707v2.

[Ch71] S. U. Chase, On inseparable Galois theory, Bull. Amer. Math. Soc. 77 (1971), 413-417.

[DM82] P. Deligne, J. Milne, Tannakian categories, pp. 101-228 in Hodge cycles, motives, and Shimura varieties, Lecture Notes in Mathematics 900, Springer-Verlag, Berlin.

[Ge64] M. Gerstenhaber, On the Galois theory of inseparable extensions, Bull. Amer. Math. Soc. 70 (1964), 561-566.

[GZ70] M. Gerstenhaber, A. Zaromp, On the Galois theory of purely inseparable field extensions, Bull. Amer. Math. Soc. 76 (1970), 1011-1014.

[Har94] D. Harbater, Abhyankar's conjecture on Galois groups over curves, Invent. Math. 117.1 (1994), pp. $1-25$.

[He71] N. Heerema, A Galois theory for inseparable field extensions, Trans. Amer. Math. Soc. 154 (1971), 193-200.

[Ja44] N. Jacobson, Galois theory of purely inseparable fields of exponent one, Amer. J. Math. 66 (1944), 645-648.

[Mo99] S. Mochizuki, The local pro-p anabelian geometry of curves, Invent. Math. 138 (1999), no. 2, 319-423.

[Mo75] J. N. Mordeson, On a Galois theory for inseparable field extensions, Trans. Amer. Math. Soc. 214 (1975), 337-347.

[Ot18] S. Otabe, On a purely inseparable analogue of the Abhyankar conjecture for affine curves, Compos. Math. 154 (2018), no. 8, 1633-1658.

[Ot19] S. Otabe, An embedding problem for finite local torsors over twisted curves (2019), available at https://arxiv.org/abs/1903.00726.

[OTZ20] S. Otabe, F. Tonini, L. Zhang, A generalized Abhyankar's conjecture for simple Lie algebras in characteristic $p>5$ (2020), available at https://arxiv.org/abs/2003.03240. 
[Pop94] F. Pop, On Grothendieck's conjecture of birational anabelian geometry, Ann. of Math. (2) 139 (1994), no. 1, 145-182.

[Pop02] F. Pop, The birational anabelian conjecture revisited, preprint (2002).

[SP21] The Stacks Project Authors, Stack Project, http://stacks.math.columbia.edu/.

[TZ19] F. Tonini, L. Zhang, Algebraic and Nori fundamental gerbes, J. Inst. Math. Jussieu 18 (2019), no. $4,855-897$.

[Uch77] K. Uchida, Isomorphisms of Galois groups of algebraic function fields, Ann. of Math. (2) 106 (1977), no. 3, 589-598.

[Zh18] L. Zhang, Nori's fundamental group over a non algebraically closed field, Ann. Sc. Norm. Super. Pisa Cl. Sci. (5) 18 (2018), no. 4, 1349-1394.

Matthieu Romagny, Institut de Recherche Mathématique de Rennes, Université Rennes 1, Campus de Beaulieu, 35042 Rennes Cedex, France

Email address: matthieu.romagny@univ-rennes1.fr

Fabio Tonini, Dipartimento di Matematica e Informatica 'Ulisse Dini', Universitá degli Studi di Firenze, Viale Morgagni, 67/A, Florence 50134, italy.

Email address: fabio.tonini@unifi.it

Lei Zhang, Department of Mathematics, The Chinese University of Hong Kong, Shatin, New Territories, Hongkong

Email address: lzhang@math.cuhk.edu.hk 